\title{
High-resolution X-ray imaging of the colliding wind shock in WR 147 (WN8(h)+B0.5V)
}

Julian M. Pittard ${ }^{1}$, Ian R. Stevens ${ }^{2}$, Peredur M. Williams ${ }^{3}$, Andrew M.T. Pollock ${ }^{4}$, Steven L. Skinner ${ }^{5}$, Michael F. Corcoran ${ }^{6,7}$, and Anthony F.J. Moffat ${ }^{8}$

${ }^{1}$ Department of Physics and Astronomy, The University of Leeds, Woodhouse Lane, Leeds LS2 9JT, UK

${ }^{2}$ School of Physics and Astronomy, University of Birmingham, Edgbaston, Birmingham B15 2TT, UK

${ }^{3}$ Institute for Astronomy, University of Edinburgh, Royal Observatory, Blackford Hill, Edinburgh EH9 $3 \mathrm{HJ}$, Scotland

${ }^{4}$ Computer \& Sci. Co. Ltd., 230 Graham Rd., Sheffield, S103GS, UK

${ }^{5}$ Center for Astrophysics and Space Astronomy, University of Colorado, UCB 389, Boulder, CO 80309, USA

${ }^{6}$ Universities Space Research Association, 7501 Forbes Blvd, Ste 206, Seabrook, MD20706, USA,

${ }^{7}$ Laboratory for High Energy Astrophysics, NASA Goddard Space Flight Center, Greenbelt, MD20771, USA

${ }^{8}$ Département de physique, Université de Montréal, C.P. 6128, Succ. Centre-Ville, QC H3C 3J7, Canada

\begin{abstract}
We present the results of a high-resolution Chandra X-ray image of the Wolf-Rayet binary system WR 147, which consists of a WN8 star and an early-type companion located 0.6 to its north. The image provides the first direct evidence for spatially extended X-ray emission in an early-type binary system, and shows that the emission peaks north of the WN8 star and close to the position of the radio bow shock. Simulated X-ray images of the wind-wind collision have a FWHM consistent with the data, but cannot account for the full spatial extension, unless emission from the stars is also included.
\end{abstract}

\section{Introduction}

Massive, early-type binaries with powerful stellar winds can generate a complex region of shock-heated plasma, with temperatures in excess of $10^{7} \mathrm{~K}$ when these winds collide (Prilutskii \& Usov 1976; Cherepashchuk 1976). However, in all previous X-ray observations, the colliding wind emission has remained spatially unresolved, and contaminated by the emission from the individual winds. Chandra provides for the first time the capability to spatially resolve the wind-wind interaction region at X-ray energies, and therefore to conclusively determine the origin of the X-ray excess in early-type binaries. WR 147 has a sufficiently great separation between the stars for this to be feasible. Previous radio, infrared and optical observations have provided unambiguous evidence of its binarity (Williams et al. 1997; Niemela et al. 1998), and together with ram balance 

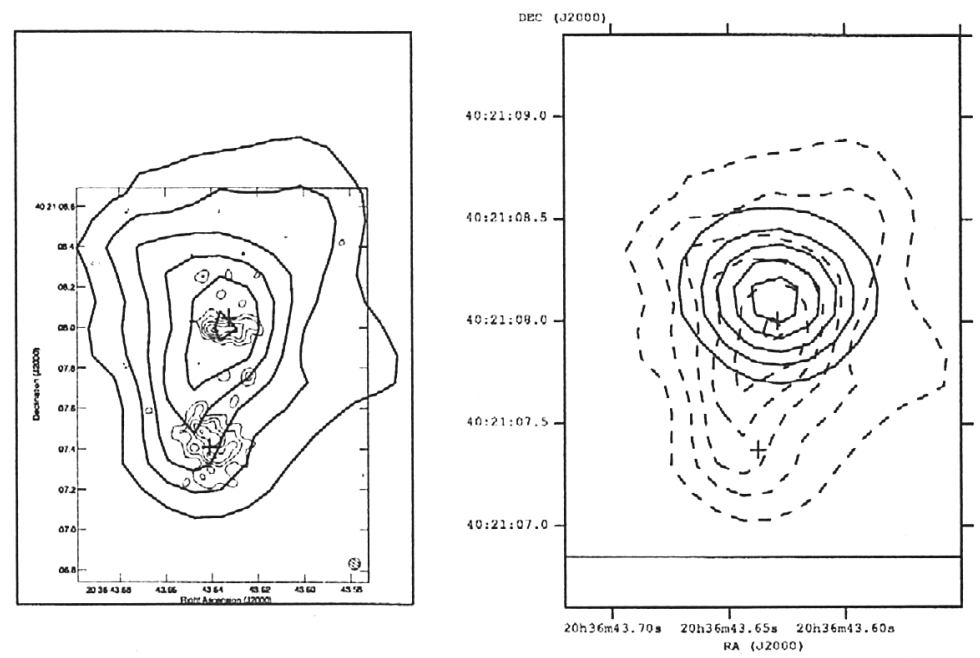

Figure 1. Left: X-ray contours (thick lines) overlaid onto radio contours from MERLIN (thin lines). The positions of the WN8 and companion star are marked with crosses. As there is some uncertainty involved in the absolute positioning of the X-ray image, we have applied a small offset $\left(-0.00965 \mathrm{~s},-0.1387^{\prime \prime}\right)$ to match the peak X-ray emission with the center of the radio bowshock. Right: a synthetic image (solid contours) of the colliding winds emission to the same scale, generated from a hydrodynamical model.

arguments and recent HST-STIS spectra (Lépine et al. 2001) support an early spectral type for the companion star. We report here on the analysis of a $5 \mathrm{ksec}$ observation of WR 147 with the Chandra-HRC-I.

\section{Results and discussion}

Figure 1 shows smoothed X-ray contours from the HRC-I overlaid onto radio contours from MERLIN. We can confidentally state that the X-ray emission peak is not co-spatial with the WN8 star, though it could be co-spatial with either the nonthermal radio peak or the northern optical component. Statistical tests reveal the $\mathrm{X}$-ray emission to be extended $(\mathrm{FWHM} \approx 0.8$, roughly double that of the PSF). We also find that models of the wind collision zone are in rough quantitative agreement, and in particular predict a FWHM consistent with that measured (Pittard et al. 2002). A deeper observation is needed to accurately measure the spatial extension, to align the X-ray and optical/radio frames, and to determine the level of contamination from the stars.

\section{References}

Cherepashchuk, A.M. 1976, Pis'ma Astron. Zh. 2, 356 (= Soviet Astron. Letters 2, 138)

Lépine, S., Wallace, D.J, Shara, M.M., et al. 2001, AJ 122, 3407

Niemela, V.S., Shara, M.M., Wallace, D.J., et al. 1998, AJ 115, 2047

Pittard, J.M., Stevens, I.R., Williams, P.M., et al. 2002, A\&A 388, 335

Prilutskii, O.F., Usov, V.V. 1976, Astron. Zh. 53, 6 (= Soviet Astron. 20, 2)

Williams, P.M., Dougherty, S.M., Davis, R.J., et al. 1997, MNRAS. 289, 10 